\title{
The Action of Group Object in A Topos
}

\author{
Tao Lu ${ }^{1, a^{*}}$ and Zhenzhen Zhu ${ }^{2, b}$ \\ ${ }^{1}$ School of Mathematics Science, HuaiBei Normal University, HuaiBei, AnHui, China \\ ${ }^{2}$ School of Mathematics Science, HuaiBei Normal University, HuaiBei, AnHui, China \\ a lutao7@live.com, b995600216@qq.com
}

Keywords: Group object; Partial object; Action.

Abstract. In this paper, based on the definition of group object, the definition of action of group object on arbitrary object in a topos is given, some equivalent characterizations are also obtained.

\section{Introduction}

Recall that a topos is a category which has finite limits and every object of has a power object. For a fixed object ${ }^{A}$ of category, the power object of ${ }^{A}$ is an object $P A$ which represents $S u b\left(_{-} \times A\right)$, so that $\left({ }_{-}, P A\right) \simeq S u b\left(\_\times A\right)$ naturally. It means that for any arrow $B^{\prime} \stackrel{f}{\rightarrow} B$, the following diagram commutes, where $\varphi$ is the natural isomorphism.

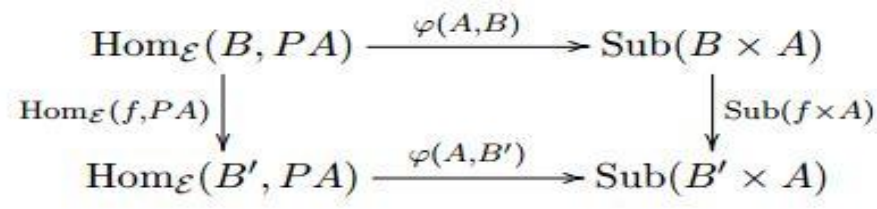

Fig. 1

As a matter of fact, the category of sheaves of sets on a topological space is a topos. In particular, the category of sets is a topos. For details of the treatment of toposes and sheaves please see [1], [2], [3], [4]. For a general background on category theory please refers to [5], [6],[7],[8],[9],[11],[12].

\section{Main results}

Throughout this paper, we work with a fixed topos, All objects mentioned belong to the topos . We begin with some definitions.

Definition 1. A group object in is an object $G$ of equipped with three arrows:

1) $e: 1 \rightarrow G$, the unit;

2) $m: G \times G \rightarrow G$, the product;

3) $i: G \rightarrow G$

And the three arrows satisfy the following diagrams.
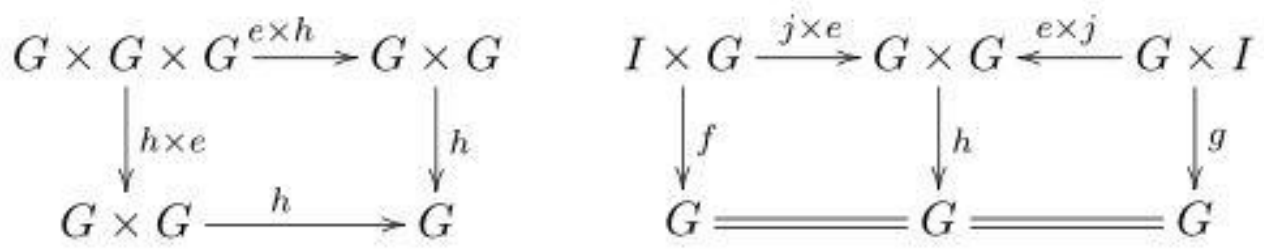

Fig. 2 


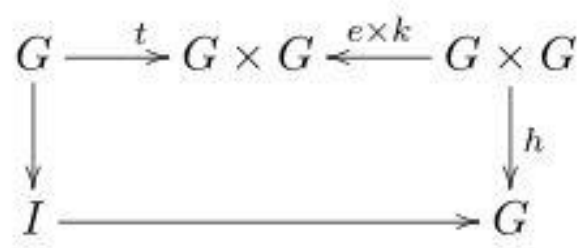

Fig. 3

In above two figures, $\mathrm{h}$ is the projective morphism and $k: G \times G \rightarrow G$ is the diagonal morphism. one can express this equivalently by the familiar identities:

$a \cdot(b \cdot c)=(a \cdot b) \cdot c ; \quad a \cdot e=e \cdot a=a$

It follows that the hom-set Hom $(X, G)$ are natural in $X$, it determines a group structure; conversely, a group structure on $\operatorname{HomE}(\mathrm{X}, \mathrm{G})$ gives the structure of an group object.

In topos , a morphism $X \stackrel{f}{\longrightarrow} G$ is regarded as a generalized element of the group objects $\mathrm{G}$, the generalized element is applied sucessfully in the patially ordered objects, please refer [8].

By the above, one can express the composite

$f g=m \circ\langle f, g\rangle: X \rightarrow G \times G \rightarrow G$

or an inverse

$f^{-1}=i \circ f: X \stackrel{f}{\longrightarrow} G \stackrel{i}{\longrightarrow} G$

Definition 2. Let $G$ be a group objects and $\Omega$ any object of . An action of $\mathrm{G}$ on $\Omega$ is a morphism $\mu=\mu_{\Omega}: G \times \Omega \rightarrow \Omega$ such that the following both diagrams commute.
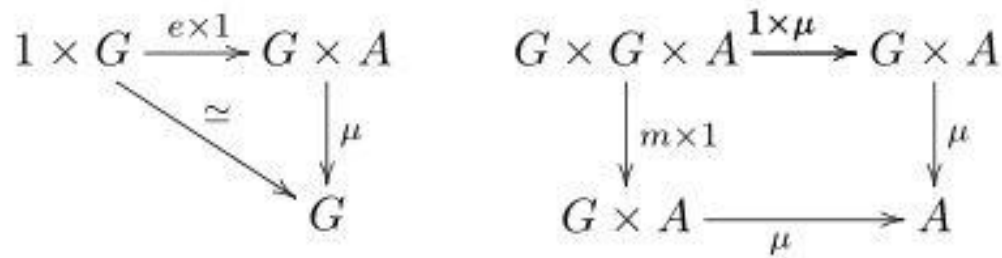

Fig. 4

This action can be denoted by a dot, as in $\mu(x, g)=x \cdot g$, for $X \stackrel{x}{\longrightarrow} \Omega, X \stackrel{g}{\longrightarrow} G$.

Definition 3. Let $G$ be any group object and $\Omega$ any object. If the action of $G$ on $\Omega$ is defined by $\alpha \cdot g=\alpha$ for all $\alpha \in \operatorname{Hom} \quad(\mathrm{X}, \Omega)$ and ${ }^{g \in} \operatorname{Hom} \quad(\mathrm{X}, \mathrm{G})$, then the action is trivial.

Definition 4. Let $G$ be any group object and $\Omega$ any object. If the identity is the only element $g \in$ Hom (X, G)such that $\alpha \cdot g=\alpha$ for all $\alpha \in \operatorname{Hom}(\mathrm{X}, \Omega)$, then the action is faithful.

In general, the kernel of an action is the set of group elements that act like 1 and "fix" all $\alpha \in$ Hom $\quad(\mathrm{X}, \Omega)$. (We say ${ }^{g}$ fixs $\alpha$ if $\alpha \cdot g=\alpha$ )

The most useful actions of finite group objects are usefully internal (in some sense) to the group

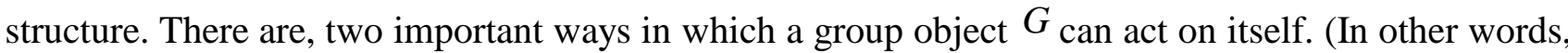
we can take $\Omega=G$.) The first of these is the regular action defined by $x \cdot g=x g$ for all $x \in$ Hom (X, $\mathrm{G})$ and $g \in$ Hom $\quad(\mathrm{X}, \mathrm{G})$. The other important action of $G$ on itself is the conjugation action, where we define $x \cdot g=x^{g}=g^{-1} x g$.

If $X \subseteq G$ is any subobject of $\mathrm{G}$ and ${ }^{g \in} \operatorname{Hom}(\mathrm{X}, \mathrm{G})$, then as usual we define the product $X g=\{x g \mid x \in X\}$. This can be used to define an action of $G$ on the set of all subsets of $G$ by setting $X \cdot g=X g$. 
Lemma 1. Let $G$ be any group object and $\Omega$ any object and $G$ act on $\Omega$. For each ${ }^{g \in}$ Hom (X, $\mathrm{G})$, define $\pi_{g}: \Omega \rightarrow \Omega$ by $(\alpha) \pi_{g}=\alpha \cdot g$. Then $\pi_{g} \in \operatorname{Sym}(\Omega)$ and the map $\theta: G \rightarrow \operatorname{Sym}(\Omega)$ defined by $\theta(g)=\pi_{g}$ is a homomorphism whose kernel is equal to the kernel of the action.

Proof. If $g, h \in \operatorname{Hom} \quad(\mathrm{X}, \mathrm{G})$ and $\alpha \in \operatorname{Hom} \quad(\mathrm{X}, \mathrm{G}))$, then

$(\alpha) \pi_{g} \pi_{h}=(\alpha \cdot g) \pi_{h}=\alpha(g h)=(\alpha) \pi_{g h}$.

and so $\pi_{g} \pi_{h}=\pi_{g h}$ for all $g, h \in G$. Also, by definition $2,(\alpha) \pi_{1}=\alpha \cdot 1=\alpha$, and so $\pi_{1}$ is the identity function $i_{\Omega}$ on $\Omega$.

Now for $g \in \operatorname{Hom}(\mathrm{X}, \mathrm{G})$, we have $\pi_{g} \pi_{g^{-1}}=\pi_{1}=\pi_{g^{-1}} \pi_{g}$, thus $\pi_{g}$ is an element of Sym $(\Omega)$.

We have $\theta(g) \theta(h)=\pi_{g} \pi_{h}=\pi_{g h}=\theta(g h)$ and $\theta_{\text {is a homomorphism. An element }} g \in G_{\text {lies in }}$

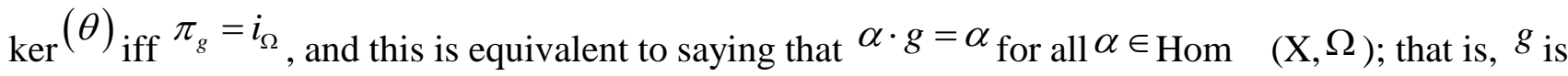
in the kernel of the action.

Group actions can also be used to produce subgroup objects. If $G$ acts on $\Omega$ and $\alpha \in \operatorname{Hom}$ (X, G), we write $G_{\alpha}=\left\{\left.g \in \operatorname{Hom}(\mathrm{X}, \mathrm{G})\right|^{\alpha \cdot g=\alpha\}}\right.$. This is called the stabilizer of $\alpha$ in Hom $(\mathrm{X}, \mathrm{G})$, and it is routine to check that $G_{\alpha}$ is always a subgroup object of $G$. We consider some examples.

Let $G$ act on itself via conjugation. If $x \in G$, then $G_{x}=\left\{g \in G \mid x^{g}=x\right\}$, and since $x^{g}=x$, we can see that the stabilizer in $G_{\text {of }} x \in \operatorname{Hom} \quad(\mathrm{X}, \mathrm{G})$ under conjugation is just $C_{G}(x)$.

We return now to the general case of a group object $G$ acting on an object $\Omega$.

Definition 4. The action is transitive if for every two elements $\alpha, \beta \in \mathrm{Hom} \quad(\mathrm{X}, \Omega)$, there exists an element $g \in$ Hom $\quad(\mathrm{X}, \mathrm{G})$ with $\alpha \cdot g=\beta$.

For instance, the regular action of $G$ and the usual action on the right cosets of a subgroup object are transitive. In general conjugation action of $G$ on itself is not transitive, since if $x, y \in$ Hom $\quad(\mathrm{X}, \mathrm{G})$ have different orders, then there can exist no $g \in \operatorname{Hom} \quad(\mathrm{X}, \mathrm{G})$ with $x^{g}=y$.

In general, if $G$ acts on $\Omega$, then the orbits of this action are the sets of the form $\{\alpha \cdot g \mid g \in$ Hom $(\mathrm{X}, \mathrm{G})\} \subseteq \Omega$.

Lemma 2. Let $G$ acts on $\Omega$. Then the orbits partition $\Omega$. This means

a. $\Omega$ is the union of the orbits and

b. any two different orbits are disjoint.

Proof. Write $O_{\alpha}=\{\alpha \cdot g \mid g \in \operatorname{Hom}(\mathrm{X}, \mathrm{G})\}$. Since $\alpha \cdot 1=\alpha$, we have $\alpha \in \mathrm{O}_{\alpha}$ and thus $\Omega=\underset{\alpha \in \Omega}{\cup} \mathrm{O}_{\alpha}$

proving part (a).

We show now that if $\gamma \in \mathrm{O}_{\alpha}$, then $\mathrm{O}_{\gamma}=\mathrm{O}_{\alpha}$.We have $\gamma=\alpha \cdot x_{\text {for some }} x \in \operatorname{Hom}(\mathrm{X}, \mathrm{G})$, and thus

$\gamma \cdot g=(\alpha \cdot x) \cdot g=\alpha \cdot x g \in \mathrm{O}_{\alpha}$

This yields $\mathrm{O}_{\gamma} \subseteq \mathrm{O}_{\alpha}$. Also, $\alpha=\gamma \cdot x^{-1}$, so that $\alpha \in \mathrm{O}_{\gamma}$ and hence the above argument yields $\mathrm{O}_{\alpha} \subseteq \mathrm{O}_{\gamma}$. We have shown that $\mathrm{O}_{\alpha}=\mathrm{O}_{\gamma}$, as claimed. 
Finally, if $\mathrm{O}_{\alpha} \cap \mathrm{O}_{\beta} \neq \varnothing$, choose $\gamma \in \mathrm{O}_{\alpha} \cap \mathrm{O}_{\beta}$. Then $\mathrm{O}_{\alpha}=\mathrm{O}_{\gamma}=\mathrm{O}_{\beta}$. And part (b) is proved.

The partition of $\Omega$ by the orbits of an action is analogous to the partition of a group by the cosets of a subgroup. This is not entirely accidental, since if $H \subseteq G$, we can let $H$ act on $G$ by right multiplication. In this case, the orbit containing $g \in G_{\text {is exactly the left cosets }} g H$.

One of the major applications of actions is for counting. The key to this is the following theorem.

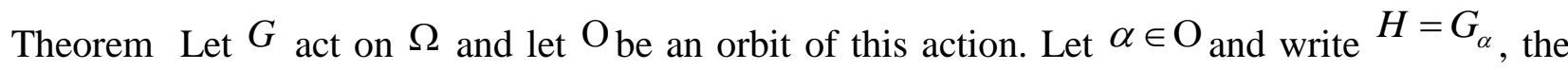
stabilizer. Then there exists a bijection $\mathrm{O} \leftrightarrow\{H x \mid x \in G\}$.

Proof. We construct a map $f: \mathrm{O} \leftrightarrow\{H x \mid x \in G\}$ as follows. If $\beta \in \mathrm{O}$, choose $x \in G$ with $\beta=\alpha \cdot x$, and set $f(\beta)=H x$. We need to check that this is well defined. In other words, if also $\beta=\alpha \cdot y$, we must establish that $H x=H y$, as required.

It is clear that $f$ maps onto $\{H x \mid x \in G\}$, since for any $x$, we have $H x=f(\alpha \cdot x)$. Finally, to show that $f$ is injective, suppose that $f(\beta)=f(\gamma)$.Then $\beta=\alpha \cdot x$ and $\gamma=\alpha \cdot y$ with $H x=H y$. This yields $y=h x$ for some $h \in H$, and hence $\gamma=\alpha \cdot y=(\alpha \cdot h) \cdot x=\alpha \cdot x=\beta$, where the third equality holds since $h \in H=G_{\alpha}$

\section{Acknowledgement}

The author would like to thank the referee for his helpful suggestions. The work is supported by the National Natural Science Foundation (Grants No.11171156)

\section{References}

[1] P.T. Johnstone, Sketches of an Elephant: a Topos Theory Compendium . vol. 2. Oxford: Oxford University Press, 2002.

[2] L.S.Mac, L.Moerdijk, Sheaves in Geometry and Logic: a first introduction to topos. Springer-Verlag, New York Berlin Heidelberg. 1992.

[3] A.Joyal, M.Tierney, An Extension of the Galois Theory of Grothendieck. Memoirs of the American Mathematical Society, 1984.

[4] P.T. Johnstone, A.Joyal, Continuous categories and exponentiable toposes. Journal of Pure and Applied Algebra, 1982, 25, pp. 255-296.

[5] W. He, Category Theory[M]. Beijing: Science Press, 2006. (in Chinese)

[6] L S. Mac., Categories for the Working Mathematician[M]. Springer-verlag, New York-Berlin, 1971.

[7] Tao Lu, Hong Lu, "On the Factorization Theorem of a Monotone Morphism in a Topos" Nonlinear Maths for Uncertainty and its Appli., AISC100, Springer-Verlag Berlin Heidelberg 2011, pp. 699-706

[8] Tao Lu, Wei He, Xijuan Wang, Galois Connections in A Topos, Journal of Mathematical Research \& Exposition, Vol. 30, No. 3, May,2010,pp.381-389 .

[9] Tao Lu, Remark on subobjects in a topos. Int. J. of Pure and Appl. Mathematics, vol 92, No. 4, 2014. 587-590. doi: 10.12732/ijpam.v92i4.13. 
[10] Xijuan Wang, Wei He. On The Heyting Algebra Objects in Topos. Journal of Mathematics, vol 31, No. 6, 2011, pp.15-20.

[11] W. He, Y.M. Liu, Steenrod Theorem for Locales, Math Proc Camb Phil Soc, vol 124, No. 2,1998, pp.305-307.

[12] W.He, T.Plewe, Directed inverse limits of spatial locales, Proceedings of the American mathematical society vol 130, No. 10 2002, pp.2811-2814. 